\title{
Very ampleness and higher syzygies for Calabi-Yau threefolds
}

\author{
Francisco Javier Gallego $^{1, \star}$, B.P. Purnaprajna ${ }^{2}$ \\ ${ }^{1}$ Dpto. de Álgebra, Facultad de Matemáticas, Universidad Complutense de Madrid, E-28040 Madrid, \\ Spain (e-mail: gallego@eucmos.sim.ucm.es) \\ 2 Department of Mathematics, University of Missouri, Columbia, MO 65211, USA \\ (e-mail: purna@math.missouri.edu)
}

To our friend K. Guruprasad

\section{Introduction}

In this article we prove results on very ampleness, projective normality and higher syzygies for Calabi-Yau threefolds.

In the first section we prove optimal results on very ampleness and projective normality for powers of ample and base-point-free line bundles. Let $X$ be a Calabi-Yau threefold and let $B$ be an ample and base-point-free line bundle on $X$. The main results of Sect. 1 can be summarized in the two following theorems (for a stronger statement of Theorem 2, see Theorem 1.7):

Theorem 1 (cf. Theorem 1.4). The line bundle $B^{\otimes 3}$ is very ample and $\left|B^{\otimes 3}\right|$ embeds $X$ as a projectively normal variety if and only if the morphism induced by $|B|$ does not map $X 2: 1$ onto $\mathbf{P}^{3}$.

Theorem 2. The line bundle $B^{\otimes 2}$ is very ample and $\left|B^{\otimes 2}\right|$ embeds $X$ as a projectively normal variety if $|B|$ does not map $X$ onto a variety of minimal degree other than $\mathbf{P}^{\mathbf{3}}$ nor maps $X 2: 1$ onto $\mathbf{P}^{\mathbf{3}}$.

A Calabi-Yau threefold is the three-dimensional version of a K3 surface and Theorems 1 and 2 are analogues of the well known results of St. Donat for K3 surfaces. Precisely, for a K3 surface $S$ and an ample and base-point-free line bundle $B$ on $S$, St. Donat proved the following (see [S-D]; see also [Ma]):

(1) $B^{\otimes 2}$ is very ample and $\left|B^{\otimes 2}\right|$ embeds $S$ as a projectively normal variety if and only if the morphism induced by $|B|$ does not map $S 2: 1$ onto $\mathbf{P}^{2}$.

(2) $B$ is very ample and $|B|$ embeds $S$ as a projectively normal variety if $|B|$ does not map $S$ onto a variety of minimal degree nor maps $X 2: 1$ onto $\mathbf{P}^{2}$.

* Partially supported by DGICYT Grant PB93-0440-C03-01 
If Fujita's conjecture is true, then $A^{\otimes 5}$ would be very ample for any ample line bundle $A$ on a Calabi-Yau threefold $X$. As corollaries of Theorems 1 and 2 and results of Ein, Lazarsfeld, Fujita and Kawamata on global generation on smooth threefolds, we obtain bounds towards Fujita's conjecture. For instance we show:

Corollary 1. Let $X$ be a smooth Calabi-Yau threefold and let $A$ be an ample line bundle such that $A^{3}>1$. Let $L=A^{\otimes n}$. If $n \geq 8$, then $L$ is very ample and $|L|$ embeds $X$ as a projectively normal variety.

For more results on very ampleness we refer the reader to Corollary 1.10.

We end Sect. 1 with a result regarding very ampleness and projective normality on Calabi-Yau fourfolds.

Section 2 is devoted to the computation of Koszul cohomology groups on Calabi-Yau threefolds. The work of Mark Green in the 80's connected Koszul cohomology with the study of equations and free resolutions of projective varieties. From our Koszul cohomology computations we obtain results regarding the equations and higher syzygies associated to powers of ample and base-point-free line bundles. We also study the Koszul property for these bundles (see Theorem 2.7). Regarding equations and higher syzygies we prove the following

Theorem 3 (cf. Theorem 2.4). Let $X$ be a Calabi-Yau threefold and let $B$ be an ample and base-point-free line bundle on $X$ such that $|B|$ does not map $X$ onto $\mathbf{P}^{3}$. If $n \geq p+2$ and $p \geq 1$, then $B^{\otimes n}$ satisfies property $N_{p}$. In particular, if $n \geq 3$, the homogeneous ideal associated to the embedding given by $\left|B^{\otimes n}\right|$ is generated by quadrics.

The parallelism between K3 surfaces and Calabi-Yau threefolds goes over to higher syzygies. In fact Theorem 3 is analogous to the following result proved by the authors in [GP1]:

Let $S$ be a $K 3$ surface and let $B$ be an ample and base-point-free line bundle on $S$ such that $|B|$ does not map $S$ onto $\mathbf{P}^{2}$. If $n \geq p+1$ and $p \geq 1$, then $B^{\otimes n}$ satisfies property $N_{p}$.

As a corollary of Theorem 3 we obtain bounds for a power of an ample line bundle to satisfy property $N_{p}$. We show precisely the following

Corollary 2 (cf. Corollary 2.8). Let $X$ be a smooth Calabi-Yau threefold and let $A$ be an ample line bundle. Let $L=A^{\otimes n}$. If $n \geq 4 p+8$, then $L$ satisfies property $N_{p}$ and the coordinate ring of the image of the embedding induced by $|L|$ is Koszul. Moreover, if $A^{3}>1$ and $n \geq 3 p+6$, then L satisfies property $N_{p}$ and the coordinate ring of $X$ is Koszul. In particular, if $n \geq 12$, or if $n \geq 9$ and $A^{3}>1$, then the ideal associated to the embedding induced by $|L|$ is generated by quadratic equations.

The article focuses on smooth Calabi-Yau threefolds for the sake of simplicity. However the arguments used also go through for Calabi-Yau threefolds with terminal singularities and for Calabi-Yau threefolds with canonical singularities. In fact Theorems 1, 2 and 3 hold for Calabi-Yau threefolds with canonical singularities. From them we recover and strengthen results by Oguiso and Peter- 
nell (see [OP]). The case of singular Calabi-Yau threefolds is dealt with in the appendix at the end of the article.

We thank Dale Cutkosky and Mohan Kumar for their encouragement as well as for useful discussions. We also thank Sheldon Katz for his encouragement and discussions regarding examples of Calabi-Yau threefolds. We are also grateful to Vladimir Maşek, who brought to our attention the work of Oguiso and Peternell, and to Frank Olaf Schreyer.

Convention. Throughout this article we work over an algebraically closed field of characteristic 0. In Sects. 1 and 2, Calabi-Yau threefolds are assumed to be smooth.

Definition. Let $X$ be a projective variety and let $L$ be a very ample line bundle on $X$. We say that $L$ is normally generated or that $L$ satisfies the property $N_{0}$, if $|L|$ embeds $X$ as a projectively normal variety. We say that $L$ is normally presented or that $L$ satisfies the property $N_{1}$ if $L$ satisfies property $N_{0}$ and, in addition, the homogeneous ideal associated to the embedding of $X$ given by $|L|$ is generated by quadratic equations. We say that $L$ satisfies the property $N_{p}$ for $p>1$, if $L$ satisfies property $N_{1}$ and the free resolution of the homogeneous ideal of $X$ is linear until the $(p-1)$ th-stage.

\section{Very ampleness and projective normality}

A Calabi-Yau threefold $X$ is a projective variety of dimension 3 with trivial canonical bundle and $H^{1}\left(\mathscr{Q}_{X}\right)=0$. In this section we study when a power of an ample and base-point-free line bundle $A$ on a Calabi-Yau threefold is very ample and when its complete linear series embeds $X$ as a projectively normal variety. We recall the following corollary of Theorem 1.3 in [GP2] which can be proven using arguments based upon Castelnuovo-Mumford regularity and Koszul cohomology:

Corollary 1.1 ([GP2], Corollary 1.6). Let $X$ be a Calabi-Yau $m$-fold, and $B$ an ample and base-point-free line bundle on $X$. If $n \geq p+m$ and $p \geq 1$ then $B^{\otimes n}$ satisfies property $N_{p}$.

Corollary 1.1 tells us in particular that if $X$ is a Calabi-Yau threefold and $n \geq 4$, then $B^{\otimes n}$ satisfies property $N_{0}$, i.e., is very ample and embeds the variety as a projectively normal variety. The main concern of this section is dealing with the case $n=2$ (Theorem 1.7) and $n=3$ (Theorem 1.4). For that purpose one has to take into account the particular properties of Calabi-Yau threefolds. The strategy to follow will be to find suitable divisors on the threefold and to translate the questions on surjectivity of multiplication maps on the threefold to questions on surjectivity of multiplication maps on the divisor. These arguments will be fruitfully repeated, eventually reaching the situation in which one confronts the question of surjectivity of multiplications maps on curves. Thus results on surjectivity of maps on curves, like [B], Proposition 2.2 and [P], Corollary 4, and 
on surfaces, like [G1], Theorem 3.9.3 for surfaces of general type (see also [C]), will be of great interest to us. Before we proceed with the statements and proofs of Theorem 1.4 and Theorem 1.7, we introduce two auxiliary tools which will be used throughout:

Observation 1.2 . Let $E, L_{1}$ and $L_{2}$ be coherent sheaves on a variety $X$. Consider the multiplication map of global sections $H^{0}(E) \otimes H^{0}\left(L_{1} \otimes L_{2}\right) \stackrel{\psi}{\rightarrow} H^{0}\left(E \otimes L_{1} \otimes L_{2}\right)$ and the maps

$$
\begin{gathered}
H^{0}(E) \otimes H^{0}\left(L_{1}\right) \stackrel{\alpha_{1}}{\longrightarrow} H^{0}\left(E \otimes L_{1}\right) \text { and } \\
H^{0}\left(E \otimes L_{1}\right) \otimes H^{0}\left(L_{2}\right) \stackrel{\alpha_{2}}{\longrightarrow} H^{0}\left(E \otimes L_{1} \otimes L_{2}\right) .
\end{gathered}
$$

If $\alpha_{1}$ and $\alpha_{2}$ are surjective then $\psi$ is also surjective.

Observation 1.3 ([GP2], Observation 2.3). Let $X$ be a regular variety (i.e, a variety such that $\left.H^{1}\left(\mathscr{O}_{X}\right)=0\right)$. Let $E$ be a vector bundle on $X$ and let $C$ be a divisor such that $L=Q_{X}(C)$ is a globally generated line bundle and $H^{1}(E \otimes$ $\left.L^{-1}\right)=0$. If the multiplication map

$$
H^{0}\left(E \otimes \mathscr{Q}_{C}\right) \otimes H^{0}\left(L \otimes \mathscr{Q}_{C}\right) \rightarrow H^{0}\left(E \otimes L \otimes \mathscr{Q}_{C}\right) \text { surjects },
$$

then the multiplication map

$$
H^{0}(E) \otimes H^{0}(L) \rightarrow H^{0}(E \otimes L) \text { also surjects. }
$$

Now we will state and prove Theorem 1.4, which give necessary and sufficient conditions for $B^{\otimes 3}$ to satisfy property $N_{0}$. Before we recall the definition of sectional genus:

Definition. Let $X$ be an irreducible normal projective variety of dimension $n$ and let $L$ be a line bundle on $X$. The sectional genus $g(L)$ of $L$, or more precisely, the sectional genus of $(X, L)$ is defined as

$$
2 g(L)-2=\left(K_{X}+(n-1) L\right) \cdot L^{n-1},
$$

where $K_{X}$ denotes the canonical divisor of $X$. Note that if $|L|$ possesses an irreducible and reduced member $S$, then the sectional genus of $(X, L)$ equals the sectional genus of $\left(S, L \otimes O_{S}\right)$ and if $X$ is reduced and irreducible of dimension 1 , then the sectional genus of $(X, L)$ is the arithmetic genus of $X$.

Theorem 1.4. Let $X$ be a Calabi-Yau threefold and let $B$ be an ample and basepoint-free line bundle. Then $B^{\otimes 3}$ is very ample and $\left|B^{\otimes 3}\right|$ embeds $X$ as a projectively normal variety except if $h^{0}(B)=4$ and the sectional genus of $B$ is 3 , in which case $B^{\otimes 3}$ is not even very ample. 
Proof. Case 1: $h^{0}(B) \geq 5$. It is enough to see that the map

$$
H^{0}\left(B^{\otimes 3+k}\right) \otimes H^{0}\left(B^{\otimes 3+l}\right) \rightarrow H^{0}\left(B^{\otimes 6+k+l}\right)
$$

surjects for all $k, l \geq 0$. By Observation 1.2 it is enough to prove a stronger statement, namely, that the map

$$
H^{0}\left(B^{\otimes 3+l}\right) \otimes H^{0}(B) \rightarrow H^{0}\left(B^{\otimes 4+l}\right)
$$

surjects for all $l \geq 0$. Castelnuovo-Mumford regularity arguments will not work if $l=0$, so we consider a smooth divisor $S \in|B|$ and the following commutative diagram:

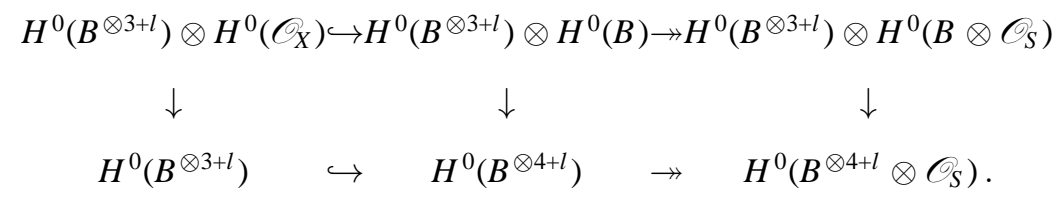

The map whose surjectivity we wish to show is the middle vertical map. The surjectivity of the left hand side vertical map is obvious. Note that $B \otimes \mathscr{O}_{S}=K_{S}$. Since $H^{1}\left(B^{\otimes 2+l}\right)=0$ for all $l$, checking the surjectivity of the right hand side reduces to checking the surjectivity of

$$
H^{0}\left(K_{S}^{\otimes 3+l}\right) \otimes H^{0}\left(K_{S}\right) \stackrel{\alpha}{\rightarrow} H^{0}\left(K_{S}^{\otimes 4+l}\right) \text { for all } l \geq 0 .
$$

To see that $\alpha$ surjects we consider now a smooth divisor $C \in\left|K_{S}\right|$. By Observation 1.3 and Kodaira vanishing, checking the surjectivity of $\alpha$ reduces to checking the surjectivity of

$$
H^{0}\left(\theta^{\otimes 3+l}\right) \otimes H^{0}(\theta) \stackrel{\beta}{\rightarrow} H^{0}\left(\theta^{\otimes 4+l}\right),
$$

where $\theta=B \otimes \mathscr{Q}_{C}$ is a theta-characteristic. We can now apply either [P], Corollary 4 or [B], Proposition 2.2 to show the surjectivity of $\beta$. For instance, to apply [P], Corollary 4, we need that either $\theta$ or $\theta^{\otimes 3+l}$ be very ample, that both $h^{0}(\theta)$ and $h^{0}\left(\theta^{\otimes 3+l}\right)$ be greater than or equal to 3 and that $\operatorname{deg} \theta^{\otimes 3+l}+\operatorname{deg} \theta$ be greater than or equal to both $3 g-3$ and $4 g-1-2 h^{1}(\theta)-2 h^{1}\left(\theta^{\otimes 3+l}\right)-\operatorname{Cliff}(C)$. The line bundle $\theta^{\otimes 3+l}$ is very ample because by Clifford's bound $g(C) \geq 5$, and the required bounds on the number of linearly independent global sections of $\theta$ and $\theta^{\otimes 3+l}$ are also satisfied since $h^{0}(B) \geq 5$. Finally, the last condition required follows from $\operatorname{deg} \theta^{\otimes 3+l}+\operatorname{deg} \theta \geq 4 g-4$ and $h^{1}(\theta) \geq 3$.

Case 2: $h^{0}(B)=4$. Let $\pi$ be the morphism induced by $|B|$. Let $C$ be as above. Since $B \otimes \mathscr{O}_{C}$ has degree $g(C)-1$ and it is the pullback of $\mathscr{Q}_{\mathbf{P}}(1)$ for a general $\mathbf{P}^{1}$ in $\mathbf{P}\left(H^{0}(B)\right)=\mathbf{P}^{3}$, the degree $n$ of $\pi$ is $g(C)-1$. In particular, $g(C) \geq 3$. If $g(C)=3, B^{\otimes 3} \otimes \mathscr{O}_{C}=K_{C} \otimes \theta$, where $\theta$ has degree 2 . Therefore the restriction of $B^{\otimes 3}$ to $C$ is not very ample. Now we treat the case $g(C) \geq 4$. It suffices to see the surjectivity of

$$
H^{0}\left(B^{\otimes 3+l}\right) \otimes H^{0}\left(B^{\otimes 3+k}\right) \rightarrow H^{0}\left(B^{\otimes 6+k+l}\right) \text { for all } l, k \geq 0 .
$$


The key case is $k=l=0$. If $l \geq 1$ or $k \geq 1$, the surjectivity of the above map follows from the arguments displayed below for the case $k=l=0$, or alternatively from Observation $1.2,[\mathrm{Mu}]$, p. 41, Theorem 2 and Kodaira vanishing Theorem. Therefore we focus our attention on the case $l=k=0$. It follows from Observation 1.2 that it is enough to check the surjectivity of

$$
\begin{gathered}
H^{0}\left(B^{\otimes 3}\right) \otimes H^{0}\left(B^{\otimes 2}\right) \stackrel{\alpha}{\rightarrow} H^{0}\left(B^{\otimes 5}\right) \\
H^{0}\left(B^{\otimes 5}\right) \otimes H^{0}(B) \stackrel{\beta}{\rightarrow} H^{0}\left(B^{\otimes 6}\right) .
\end{gathered}
$$

The map $\beta$ surjects by [Mu], Theorem 2 and Kodaira vanishing Theorem. Note that we cannot use Observation 1.2 again in order to prove the surjectivity of $\alpha$, because the map $H^{0}\left(B^{\otimes 3}\right) \otimes H^{0}(B) \rightarrow H^{0}\left(B^{\otimes 4}\right)$ is actually non surjective, for otherwise the map

$$
H^{0}\left(K_{C} \otimes \theta\right) \otimes H^{0}(\theta) \rightarrow H^{0}\left(K_{C}^{\otimes 2}\right)
$$

would also surject, but this is false by base-point-free pencil trick. Instead the surjectivity of $\alpha$ will follow from the surjectivity of $\gamma$ and $\delta$ in the diagram

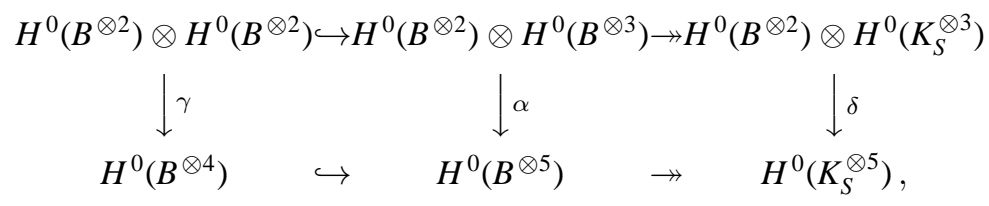

obtained from the sequence

$$
0 \longrightarrow B^{*} \longrightarrow \mathscr{O}_{X} \longrightarrow \mathscr{O}_{S} \longrightarrow 0 .
$$

To see the surjectivity of $\gamma$ we construct yet another two similar diagrams arising from (1.4.1). Since $H^{1}\left(B^{\otimes r}\right)=0$ for all $r \geq 0$, checking the surjectivity of $\gamma$ reduces to seeing the surjectivity of

$$
\begin{aligned}
& H^{0}\left(K_{S}^{\otimes 2}\right) \otimes H^{0}\left(K_{S}^{\otimes 2}\right) \stackrel{\epsilon}{\rightarrow} H^{0}\left(K_{S}^{\otimes 4}\right) \\
& H^{0}\left(K_{S}^{\otimes 2}\right) \otimes H^{0}\left(K_{S}\right) \stackrel{\eta}{\rightarrow} H^{0}\left(K_{S}^{\otimes 3}\right) .
\end{aligned}
$$

On the other hand in order to see the surjectivity of $\delta$, again by the vanishing of $H^{1}\left(B^{\otimes r}\right)$ it is enough to check the surjectivity of

$$
H^{0}\left(K_{S}^{\otimes 3}\right) \otimes H^{0}\left(K_{S}^{\otimes 2}\right) \stackrel{\varphi}{\rightarrow} H^{0}\left(K_{S}^{\otimes 5}\right) .
$$

For the surjectivity of $\epsilon, \eta$ and $\varphi$ we build commutative diagrams like the one above, now upon the sequence

$$
0 \rightarrow K_{S}^{*} \rightarrow \mathscr{C}_{S} \rightarrow \mathscr{C}_{C} \rightarrow 0 .
$$

For instance, to see the surjectivity of $\epsilon$ we would write: 


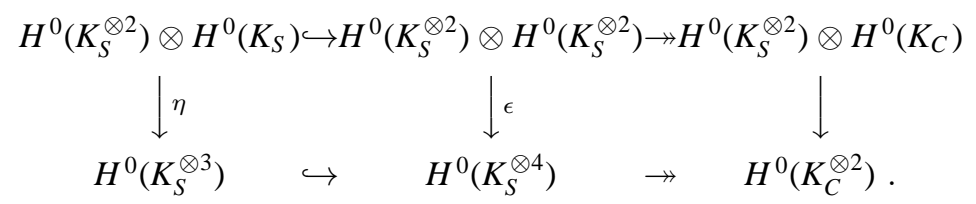

Since $H^{1}\left(K_{S}^{\otimes r}\right)=0$ for all $r \geq 0$, the surjectivity of $\epsilon, \eta$ and $\varphi$ will follow from the surjectivity of the maps

$$
\begin{gathered}
H^{0}\left(K_{C}\right) \otimes H^{0}\left(K_{C}\right) \rightarrow H^{0}\left(K_{C}^{\otimes 2}\right) \\
H^{0}\left(K_{C}\right) \otimes H^{0}(\theta) \rightarrow H^{0}\left(K_{C} \otimes \theta\right) \text { and } \\
H^{0}\left(K_{C}\right) \otimes H^{0}\left(K_{C} \otimes \theta\right) \rightarrow H^{0}\left(K_{C}^{\otimes 2} \otimes \theta\right) .
\end{gathered}
$$

Recall that $g(C) \geq 4$, therefore $C$ cannot be hyperelliptic as $\left|B \otimes \mathscr{O}_{C}\right|$ is a base-point-free pencil of degree $g-1$. Thus the first map above is surjective by Nöther's theorem. For the second, recall that $\theta$ is a theta-characteristic, that it is base-point-free and that $h^{0}(\theta)=2$. Thus the surjectivity follows from the base-point-free pencil trick. Finally the third one follows from [P], Corollary 4.

Remark 1.4.2. The same arguments used in the proof of Theorem 1.4 show that given any nef line bundle $N$, the line bundle $B^{\otimes 3} \otimes N$ is very ample and embeds $X$ as a projectively normal variety, if $h^{0}(B) \geq 5$. Moreover this statement holds if $X$ is a threefold with numerically trivial canonical bundle and $h^{1}\left(\mathscr{C}_{X}\right)=0$.

We show now by means of an example that there indeed exist ample and base-point free line bundles with four linearly independent global sections and sectional genus 3 :

Example 1.5. Let $X$ be the double cover of $\mathbf{P}^{3}$ ramified along a smooth degree 8 surface and let $B$ be the pullback of $\mathscr{C}_{\mathbf{p}}(1)$. The threefold $X$ is Calabi-Yau, $h^{0}(B)=4$ and the sectional genus of $B$ is 3 .

We now want to know when $B^{\otimes 2}$ is normally generated. In the study we carry on we will use a theorem by M. Green. To apply this theorem we will require the image of the morphism $\pi$ induced by $|B|$ not to be a variety of minimal degree. For that reason it is interesting to study the characteristics of $\pi$ when its image is a variety of minimal degree. In the first part of the following proposition we obtain a bound on the degree of $\pi$ and in the second part we classify the different kinds of varieties of minimal degree which can appear and the structure of the Calabi-Yau threefold $X$.

Proposition 1.6. Let X be a (smooth) Calabi-Yau threefold, let $\pi$ be the morphism induced by the complete linear series of an ample and base-point-free line bundle $B$ on $X$ with $h^{0}(B)=r+1$, and let $n$ be the degree of $\pi$. If the image of $X$ by $\pi$ is a variety $Y$ of minimal degree, then $n \leq \frac{6 r+6}{r-2}$ and one of the following occurs:

1. $Y=\mathbf{P}^{3}$.

2. $Y$ is a smooth quadric hypersurface in $\mathbf{P}^{4}$. 
3. $Y$ is a smooth rational normal scroll of dimension 3 in $\mathbf{P}^{\mathbf{5}}$.

4. $Y$ is a smooth rational normal scroll in $\mathbf{P}^{\mathbf{r}}, r \geq 6$, the degree $n$ of $\pi$ is 2 and $X$ is fibered over $\mathbf{P}^{\mathbf{1}}$ with a smooth $K 3$ surface as a general fiber. The restriction of $B$ to the general fiber of $X$ is hyperelliptic, with sectional genus 2 , and its complete linear series maps the fiber $2: 1$ onto a general fiber of the scroll.

5. $Y$ is a smooth rational normal scroll in $\mathbf{P r}^{\mathbf{r}}, r \geq 6$, the degree $n$ of $\pi$ is 6 and $X$ is fibered over $\mathbf{P}^{\mathbf{1}}$ with a smooth Abelian surface as a general fiber. The restriction of $B$ to the general fiber of $X$ is a $(1,3)$ polarization, and its complete linear series maps the fiber $6: 1$ onto a general fiber of the scroll.

6. $Y$ is a singular threefold of minimal degree which is either a cone over a conic in $\mathbf{P}^{\mathbf{2}}$, a twisted cubic in $\mathbf{P}^{\mathbf{3}}$, or a Veronese surface.

Proof. First we prove that the inequality

$$
n \leq \frac{6 r+6}{r-2} \quad(*)
$$

holds if $Y$ is a variety of minimal degree. By Riemann-Roch and since $c_{2}(X) \cdot B \geq$ 0 (cf. [Mi], Theorem 1.1), $r+1=h^{0}(B) \geq \frac{1}{6} B^{3}=\frac{1}{6} n(r-2)$, so we obtain (*).

Now we describe all the possible types of varieties of minimal degree that may occur. The variety $Y$ should be either $\mathbf{P}^{\mathbf{3}}$, a smooth quadric hypersurface in $\mathbf{P}^{\mathbf{4}}$, a singular 3-dimensional rational normal scroll in $\mathbf{P}^{\mathbf{4}}$, a (possibly singular) 3dimensional rational normal scroll in $\mathbf{P}^{\mathbf{r}}, r \geq 5$ or a cone in $\mathbf{P}^{\mathbf{6}}$ over a Veronese surface in $\mathbf{P}^{\mathbf{5}}$. We see now that $Y$ cannot be a 3-dimensional rational normal scroll singular along a single point. In that case, in the notation of $[\mathrm{EH}], Y=$ $S(0, b, c)$ with $0<b \leq c$. By this we mean that $Y$ is the image of the projective bundle $Z=\mathbf{P}(\mathscr{E})$ over $\mathbf{P}^{1}$ by the morphism $\psi$ induced by $\left|\mathscr{Q}_{\mathbf{P}(\mathscr{E})}(1)\right|$, where $E=\odot \oplus \odot(b) \oplus \mathscr{C}(c)$. Thus $\psi$ would be a small resolution for $Y$. Then it would follow that $X$ could also be obtained by performing small contractions on $\left(X \times_{Y} Z\right)_{\text {red }}$ and $X$ would not be smooth.

We show now that if $Y$ is a cone over a rational normal curve then $r=4$ or 5. Let $G$ be the inverse image of a general plane $F$ in $Y$. Then $B^{2} \cdot G=n$. On the other hand $B$ can be written as the pullback of $(r-2) F$, hence $G$ turns out to be an ample divisor on $X$ and $n=(r-2)^{2} G^{3} \geq(r-2)^{2}$. By (*), we get that $(r-2)^{3}-6 r-6 \leq 0$. When $r \geq 4$, the previous inequality is only satisfied for $r=4,5$.

We complete now the proof of the proposition by studying the case when $Y$ is a smooth rational normal scroll and $r \geq 6$. In such a case $Y$ is fibered over $\mathbf{P}^{\mathbf{1}}$ and so is $X$. Let $\varphi$ be the projection from $Y$ to $\mathbf{P}^{\mathbf{1}}$. Let us denote by $F$ a general fiber of $\varphi$, and let $G$ be a general fiber of $\varphi \circ \pi$. We consider the following sequence:

$$
0 \longrightarrow H^{0}(B(-G)) \longrightarrow H^{0}(B) \longrightarrow H^{0}\left(B \otimes \bigodot_{G}\right) \longrightarrow H^{1}(B(-G)) \longrightarrow 0 .
$$

Since $r \geq 6$ and $Y$ is smooth, $Y=S(a, b, c)$ (i.e., $Y$ is isomorphic to $\mathbf{P}(\mathscr{E})$, where $\mathscr{E}=\mathscr{O}(a) \oplus \mathscr{O}(b) \oplus \mathscr{O}(c)$, mapped in projective space by $\left.\left|\mathscr{Q}_{\mathbf{P}(\mathscr{C})}(1)\right|\right)$, 
with $a \leq b \leq c, a \geq 1$, and $c \geq 2$. Let $H$ be the restriction of $\mathscr{C}_{\mathbf{p r}}(1)$ to $Y$. Then $H(-F)$ is big and globally generated, in particular, big and nef, and $\pi$ being finite, so is $B(-G)$. Thus by Kawamata-Viehweg, $H^{1}(B(-G))=0$, which implies that $\left|B \otimes \mathscr{C}_{G}\right|$ maps $G$ onto $\mathbf{P}^{2}$. Since $B$ is ample, $G$ is connected, and by Bertini, smooth. Then, by adjunction, $G$ is either a smooth K3 surface or a smooth Abelian surface. If $G$ is a smooth K3 surface, then $\left(G, B \otimes \mathscr{O}_{G}\right)$ is a genus 2, hyperelliptic polarized K3 surface, $\pi \mid G$ is $2: 1$ and so is $\pi$. If $G$ is a smooth Abelian surface, then $B \otimes \mathscr{C}_{G}$ is a $(1,3)$-polarization, hence $\pi \mid G$ has degree 6 and so has $\pi$.

We return our attention to the normal generation of $B^{\otimes 2}$ :

Theorem 1.7. Let $X$ be a (smooth) Calabi-Yau threefold and let $B$ be an ample and base-point-free line bundle on $X$.

1. If the image of $X$ by the morphism $\pi$ induced by the complete linear series of $B$ is not a variety of minimal degree, i.e., is not one of the six cases in the list of Proposition 1.6, then $B^{\otimes 2}$ is very ample and embeds $X$ as a projectively normal variety.

2. In case 1 of Proposition 1.6, $B{ }^{\otimes 2}$ is very ample and embeds $X$ as a projectively normal variety if and only if the sectional genus of $B$ is not 3 .

3. If the degree $n$ of $\pi$ equals 2 (for instance, in case 4 of Proposition 1.6), $B^{\otimes 2}$ is not even very ample.

Proof. We prove (1) first. By hypothesis, $h^{0}(B) \geq 5$ and the image of $X$ by the morphism induced by $|B|$ is not a variety of minimal degree. We want to prove that $B^{\otimes 2}$ satisfies property $N_{0}$. We prove instead a more general statement, namely, we show that the multiplication map

$$
H^{0}\left(B^{\otimes l+2}\right) \otimes H^{0}\left(B^{\otimes 2}\right) \longrightarrow H^{0}\left(B^{\otimes l+4}\right)
$$

surjects for all $l \geq 0$. From Observation 1.2 it follows that it suffices to have the surjectivity of

$$
H^{0}\left(B^{\otimes l+2}\right) \otimes H^{0}(B) \stackrel{\alpha}{\rightarrow} H^{0}\left(B^{\otimes l+3}\right)
$$

for all $l \geq 0$. The crucial cases are $l=0,1$. If $l \geq 2$, the surjectivity of $\alpha$ can be obtained from the same arguments used below for $l=0,1$, or from Kodaira vanishing and $[\mathrm{Mu}]$, Theorem 2 . Case $l=1$ was already dealt with in the proof of Theorem 1.4. Thus we focus on case $l=0$, i.e., on the surjectivity of

$$
H^{0}\left(B^{\otimes 2}\right) \otimes H^{0}(B) \stackrel{\beta}{\rightarrow} H^{0}\left(B^{\otimes 3}\right) .
$$

We first use Observation 1.3. Since $H^{1}(B)=0$ and by adjunction $B \otimes \mathscr{O}_{S}=K_{S}$, it is enough to prove the surjectivity of

$$
H^{0}\left(K_{S}\right) \otimes H^{0}\left(K_{S}^{\otimes 2}\right) \stackrel{\delta}{\rightarrow} H^{0}\left(K_{S}^{\otimes 3}\right) .
$$


Since the image of $S$ under the morphism defined by $\left|K_{S}\right|$ is not a surface of minimal degree, $h^{0}\left(K_{S}\right)=h^{0}(B)-1 \geq 4$, and $H^{1}\left(\mathscr{O}_{S}\right)=0$, by [G1], Theorem 3.9.3, the map $\delta$ surjects. of

We prove now (2). Recall that $h^{0}(B)=4$. We want to show the surjectivity

$$
H^{0}\left(B^{\otimes 2 l}\right) \otimes H^{0}\left(B^{\otimes 2}\right) \stackrel{\alpha}{\rightarrow} H^{0}\left(B^{\otimes 2 l+2}\right)
$$

if the sectional genus of $B$ is greater than 3. If $l=1$, the surjectivity of $\alpha$ was shown in the proof of Theorem 1.4. If $l \geq 2$, the surjectivity of $\alpha$ follows from the same arguments or alternatively from Observation 1.2, Kodaira vanishing and $[\mathrm{Mu}]$, Theorem 2. On the other hand, if the sectional genus of $B$ is 3 , the morphism induced by $|B|$ is a $2: 1$ cover of $\mathbf{P}^{3}$, hence a general curve $C$ in $B \otimes O_{S}$, where $S$ is a general divisor in $|B|$, is a hyperelliptic curve. Therefore $B^{\otimes 2} \otimes \mathscr{C}_{C}=K_{C}$ is not very ample.

Finally we prove (3). Since now the morphism induced by $|B|$ is a $2: 1$ cover of a rational normal scroll and $C$ is again hyperelliptic, then $B^{\otimes 2}$ cannot be very ample.

Looking at Theorem 1.7 it can be seen that the hyperellipticity of $C$ determines in many cases whether $B^{\otimes 2}$ satisfies the property $N_{0}$ or not. For instance, the fact of $C$ being hyperelliptic forces the image of $X$ by $|B|$ to be a variety of minimal degree. We also have this

Corollary 1.8. Let $X$ be a Calabi-Yau threefold and let $B$ be an ample and basepoint-free line bundle on $X$. If $h^{0}(B)=4$ or if $(X, B)$ is of type 2 (i.e., the morphism induced by $|B|$ is generically $2: 1$ onto its image), $B{ }^{\otimes 2}$ satisfies the property $N_{0}$ if and only if there exists $S \in|B|$ and a smooth curve $C \in\left|B \otimes \mathscr{O}_{S}\right|$ which is non-hyperelliptic.

All the above motivates the following

Conjecture 1.9. Let $X$ be a Calabi-Yau threefold and let $B$ be an ample and base-point-free line bundle. Then $B^{\otimes 2}$ embeds $X$ as a projectively normal variety if and only if there is a smooth non-hyperelliptic curve $C$ in $\left|B \otimes O_{S}\right|$ for some $S \in|B|$.

Theorems 1.4 and 1.7 combined with results on global generation of powers of ample line bundles, such as Ein and Lazarsfeld's (cf. [EL2]), Fujita's (cf. [F]) and Kawamata's (cf. $[\mathrm{K}]$ ), yield the following

Corollary 1.10. Let $X$ be a (smooth) Calabi-Yau threefold and let $A$ be an ample line bundle. If $A^{3}>1$, then $A^{\otimes 6}$ and $A^{\otimes n}$ satisfy property $N_{0}$ for all $n \geq 8$. If $A^{3}=1$, then $A^{\otimes 8}$ and $A^{\otimes n}$ satisfy property $N_{0}$ for all $n \geq 10$.

Proof. The line bundle $A^{\otimes m}$ is base-point-free if $m \geq 4$ (cf. [EL2]) and, if $A^{3}>1$ and $m \geq 3$, then $A^{\otimes m}$ is base-point-free (cf. [F]). We take as $B$ one of these line bundles $A^{\otimes m}$. By Riemann-Roch, $h^{0}(B) \geq \frac{1}{6} B^{3} \geq 9$. Then it follows from Proposition 1.6 that $|B|$ does not map $X$ onto a variety of minimal degree, except 
maybe one in the cases (4) or (5) in the proposition. This cannot occur either since $B$ is at least three times an ample divisor, which contradicts the descriptions given in Proposition 1.6. Therefore $|B|$ does not map $X$ onto a variety of minimal degree, and the statement follows from Theorem 1.4, Remark 1.4.2 and Theorem 1.7 , except when $A^{3}=1$ and $n=11$. In that case the result follows from the fact $A^{\otimes 5}$ separates points by Theorem 1 in [OP] and by [G2], Theorem 4 .

To end this section we prove a result regarding very ampleness and projective normality on Calabi-Yau fourfolds. Recall that Corollary 1.1 tells among other things that if $X$ is a smooth Calabi-Yau fourfold and $B$ is an ample and basepoint-free line bundle, then $B^{\otimes n}$ satisfies property $N_{p}$ if $n \geq p+4$ and $p \geq 1$. Therefore if $n \geq 5, B^{\otimes n}$ satisfies property $N_{1}$, and in particular, $B^{\otimes n}$ is very ample and $\left|B^{\otimes n}\right|$ embeds $X$ as a projectively normal variety. In the following theorem we prove that the above holds for $B^{\otimes 4}$ under certain conditions on $B$.

Theorem 1.11. Let $X$ be a smooth Calabi-Yau fourfold and let $B$ be an ample and base-point-free line bundle such that the morphism induced by $|B|$ is birational onto the image and $h^{0}(B) \geq 7$. Then $B^{\otimes 4}$ is very ample and $\left|B^{\otimes 4}\right|$ embeds $X$ as a projectively normal variety.

Proof. From Observation 1.2 it follows that it suffices to prove the surjectivity of

$$
H^{0}\left(B^{\otimes n}\right) \otimes H^{0}(B) \longrightarrow H^{0}\left(B^{\otimes n+1}\right)
$$

for all $n \geq 4$. When $n \geq 5$, this follows from [Mu], Theorem 2 and Kodaira vanishing theorem. If $n=4$, we argue like in the proofs of Theorems 1.4 and 1.7. We consider a smooth curve $C$ obtained by iteratively taking hyperplane sections in $|B|$. Then we use Observation 1.3 and since $B \otimes 4 \otimes \mathscr{C}_{C}=K_{C}$, the problem is eventually reduced to checking the surjectivity of the following map on $C$,

$$
H^{0}\left(K_{C}\right) \otimes H^{0}(L) \stackrel{\alpha}{\rightarrow} H^{0}\left(K_{C} \otimes L\right),
$$

where $L=B \otimes \mathscr{O}_{C}$. The line bundle $L$ is ample, base-point-free, $|L|$ induces a birational morphism from $C$ onto its image, and $h^{0}(L) \geq 4$, thus the surjectivity of $\alpha$ follows from a theorem of Castelnuovo (cf. [ACGH], page 151) which states that the map $S^{n} H^{0}(L) \otimes H^{0}\left(K_{C}\right) \longrightarrow H^{0}\left(K_{C} \otimes L^{\otimes n}\right)$ surjects for all $n \geq 0$ under the conditions satisfied by $L$.

\section{Normal presentation, Koszul rings and higher syzygies}

The purpose of this section is to compute Koszul cohomology groups on CalabiYau threefolds and to apply this computation to the study of the ring, equations and free resolution of those threefolds. The connection between Koszul cohomology and syzygies was developed by Green (see [G2]; for a particularly gentle introduction to the subject see also [L]). We present now the statement we need for our purposes. Let $X$ be a projective variety, and let $F$ be a globally generated vector bundle on $X$. We define the bundle $M_{F}$ as follows: 


$$
0 \rightarrow M_{F} \rightarrow H^{0}(F) \otimes \mathscr{Q}_{X} \rightarrow F \rightarrow 0 .
$$

If $L$ is an ample line bundle on $X$ such that all its positive powers are nonspecial there exists the following characterization of property $N_{p}$ :

Theorem 2.2. Let $L$ be an ample, globally generated line bundle on a variety $X$. If the cohomology group $H^{1}\left(\bigwedge^{p^{\prime}+1} M_{L} \otimes L^{\otimes s}\right)$ vanishes for all $0 \leq p^{\prime} \leq p$ and all $s \geq 1$, then $L$ satisfies the property $N_{p}$. If in addition $H^{1}\left(L^{\otimes r}\right)=0$, for all $r \geq 1$, then the above is a necessary and sufficient condition for $L$ to satisfy property $N_{p}$.

We use this characterization to prove our results on syzygies. For the proof of it we refer to [EL1], Sect. 1. Since we are working over an algebraically closed field of characteristic 0 , for our proofs of higher syzygies results we will check the vanishings of $H^{1}\left(M_{L}^{\otimes p^{\prime}+1} \otimes L^{\otimes s}\right)$ rather than see directly the vanishings of $H^{1}\left(\bigwedge^{p^{\prime}+1} M_{L} \otimes L^{\otimes s}\right)$.

Before we state the main theorem of this section we state the following lemma (for the proof, see [GP2], Lemma 2.9):

Lemma 2.3. Let $X$ be a projective variety, let $q$ be a nonnegative integer and let $F$ be a base-point-free line bundle on $X$. Let $Q$ be an effective line bundle on $X$ and let $\mathfrak{q}$ be a reduced and irreducible member of $|Q|$. Let $R$ be a line bundle and $G$ a sheaf on $X$ such that

1. $H^{1}\left(F \otimes Q^{*}\right)=0$,

2. $H^{0}\left(M_{\left(F \otimes \mathscr{C}_{\mathfrak{q}}\right)}^{\otimes q^{\prime}} \otimes R \otimes \mathscr{C}_{\mathfrak{q}}\right) \otimes H^{0}(G) \rightarrow H^{0}\left(M_{\left(F \otimes \mathscr{C}_{\mathfrak{q}}\right)}^{\otimes q^{\prime}} \otimes R \otimes G \otimes \mathscr{C}_{\mathfrak{q}}\right)$ surjects for all $0 \leq q^{\prime} \leq q$.

Then, for all $0 \leq q^{\prime \prime} \leq q$ and all $0 \leq k^{\prime} \leq q^{\prime \prime}$,

$H^{0}\left(M_{F}^{\otimes k} \otimes M_{\left(F \otimes \mathscr{C}_{\mathfrak{q}}\right)}^{\otimes q^{\prime \prime}-k} \otimes R \otimes \mathscr{C}_{\mathfrak{q}}\right) \otimes H^{0}(G) \rightarrow H^{0}\left(M_{F}^{\otimes k} \otimes M_{\left(F \otimes \mathcal{C}_{\mathfrak{q}}\right)}^{\otimes q^{\prime \prime}-k} \otimes G \otimes R \otimes \mathscr{C}_{\mathfrak{q}}\right)$

surjects.

We are now ready the state the following

Theorem 2.4. Let $X$ be a Calabi-Yau threefold. Let $B$ be an ample and basepoint-free line bundle with $h^{0}(B) \geq 5$. Let $L=B^{\otimes p+2+k}$ and $L^{\prime}=B^{\otimes p+2+l}$. If $k, l \geq 0$ and $p \geq 1$, then $H^{1}\left(M_{L}^{\otimes p+1} \otimes L^{\prime}\right)=0$ and L satisfies property $N_{p}$.

Proof. The proof is by induction on $p$. The most important step is $p=1$. Consider the sequence

$$
\begin{aligned}
& H^{0}\left(M_{L} \otimes L^{\prime}\right) \otimes H^{0}(L) \stackrel{\alpha}{\rightarrow} H^{0}\left(M_{L} \otimes L^{\prime} \otimes L\right) \\
& \rightarrow H^{1}\left(M_{L}^{\otimes 2} \otimes L^{\prime}\right) \rightarrow H^{1}\left(M_{L} \otimes L^{\prime}\right) \otimes H^{0}(L) .
\end{aligned}
$$

The last term of the sequence vanishes by Theorem 1.4, so it suffices to prove the surjectivity of $\alpha$. For that we use Observation 1.2. We see therefore that it is enough to show that 


$$
H^{0}\left(M_{L} \otimes L^{\prime}\right) \otimes H^{0}(B) \stackrel{\beta}{\rightarrow} H^{0}\left(M_{L} \otimes L^{\prime} \otimes B\right)
$$

surjects. Let $S$ be a smooth divisor in $|B|$. The cohomology group $H^{1}\left(M_{L} \otimes B\right)$ vanishes because the map $\alpha$ surjects, as shown in the proof of Theorem 1.4. Thus by Observation 1.3 it is enough to show the surjectivity of

$$
H^{0}\left(M_{L} \otimes B \otimes \mathscr{C}_{S}\right) \otimes H^{0}\left(B \otimes \mathscr{C}_{S}\right) \stackrel{\gamma}{\rightarrow} H^{0}\left(M_{L} \otimes B^{\otimes 2} \otimes \mathscr{C}_{S}\right) .
$$

Applying now Lemma 2.3, we conclude that it suffices to see the surjectivity of

$$
H^{0}\left(M_{K_{S}^{\otimes m}} \otimes K_{S}^{\otimes n}\right) \otimes H^{0}\left(K_{S}\right) \stackrel{\delta}{\rightarrow} H^{0}\left(M_{K_{S}^{\otimes m}} \otimes K_{S}^{\otimes n+1}\right),
$$

for all $m, n \geq 3$. Let $C$ be a smooth curve in $\left|K_{S}\right|$ and set $G=K_{S}^{\otimes m} \otimes \mathscr{Q}_{C}$ and $G^{\prime}=K_{S}^{\otimes n} \otimes \mathscr{O}_{C}$. We apply Observation 1.3 and Lemma 2.3. To apply Lemma 2.3 we need to see that

$$
\begin{gathered}
H^{0}\left(G^{\prime}\right) \otimes H^{0}(\theta) \longrightarrow H^{0}\left(G^{\prime} \otimes \theta\right) \text { and } \\
H^{0}\left(M_{G} \otimes G^{\prime}\right) \otimes H^{0}(\theta) \longrightarrow H^{0}\left(M_{G} \otimes G^{\prime} \otimes \theta\right)
\end{gathered}
$$

surject, where $\theta=B \otimes \mathscr{O}_{C}$ is a theta-characteristic. To see the surjectivity of the first map, note that $\operatorname{deg}\left(G^{\prime} \otimes \mathscr{O}_{C}\right)+\operatorname{deg} \theta \geq 4 g(C)-4$. Since $h^{0}(B) \geq 5$, then $h^{1}(\theta) \geq 3$, so the surjectivity follows by [B], Proposition 2.2 or [P], Corollary 4. To see that the second map surjects, note that $K_{S}^{2} \geq 4$ by Nöther's inequality, and therefore, $\operatorname{deg} G \geq 3 g(C)-3 \geq 2 g(C)+2$. Thus $M_{G} \otimes G^{\prime}$ is semistable by [B], Theorem 1.12. We see now that the slope of $M_{G} \otimes G^{\prime}$ is bigger than $2 g(C)$. Since $H^{1}(G)=0$,

$$
\mu\left(M_{G}\right)=\frac{-\operatorname{deg} G}{\operatorname{deg} G-g(C)},
$$

therefore

$$
\mu\left(M_{G} \otimes G^{\prime}\right)=\frac{-\operatorname{deg} G}{\operatorname{deg} G-g(C)}+3 g(C)-3 \geq \frac{-\operatorname{deg} G}{\operatorname{deg} G-g(C)}+2 g(C)+2,
$$

and the last term of the above sequence of inequalities is bigger than or equal to $2 g(C)+1$. On the other hand

$$
\begin{aligned}
\mu\left(M_{G} \otimes G^{\prime}\right) & =\frac{-\operatorname{deg} G}{\operatorname{deg} G-g(C)}+3 g(C)-3 \\
& >3 g(C)-5 \\
& \geq 2 g(C)+2 g(C)-\operatorname{deg}(\theta)-2 h^{1}(\theta) .
\end{aligned}
$$

Thus the desired surjectivity follows from [B], Proposition 2.2.

For $p>1$, we write the sequence:

$$
\begin{aligned}
& H^{0}\left(M_{L}^{\otimes p} \otimes L^{\prime}\right) \otimes H^{0}(L) \stackrel{\epsilon}{\rightarrow} H^{0}\left(M_{L} \otimes L^{\prime} \otimes L\right) \\
\rightarrow & H^{1}\left(M_{L}^{\otimes p+1} \otimes L^{\prime}\right) \rightarrow H^{1}\left(M_{L} \otimes L^{\prime}\right) \otimes H^{0}(L) .
\end{aligned}
$$

The group $H^{1}\left(M_{L}^{\otimes p} \otimes L^{\prime}\right)$ vanishes by induction hypothesis. By Observation 1.2 we only need to show that 


$$
H^{0}\left(M_{L}^{\otimes p} \otimes L^{\prime}\right) \otimes H^{0}(B) \stackrel{\eta}{\rightarrow} H^{0}\left(M_{L} \otimes L^{\prime} \otimes B\right)
$$

surjects. This follows arguing similarly as in the proof of the surjectivity of $\beta$, using Observation 1.3, Lemma 2.3 to reduce the problem to checking the surjectivity of multiplication maps on $S \in|B|$ first and to checking the surjectivity of multiplication maps on $C \in\left|K_{S}\right|$ eventually, and once we are arguing on $C$, the result follows from [B], Proposition 2.2. We can argue alternatively by induction to see that $\eta$ surjects. Indeed, applying [Mu], Theorem 2, the vanishing of $H^{1}\left(M_{L}^{\otimes p} \otimes L^{\prime} \otimes B^{*}\right)$ follows by induction and the other two vanishings required follow from chasing cohomology sequences arising from (2.1) and again using induction.

Finally, the fact that $L$ satisfies property $N_{p}$ follows from the vanishings just proven, Theorem 1.4 and Theorem 2.2.

Remark 2.4.1. Theorem 2.4 holds for $X$ threefold with $K_{X} \equiv 0$ and $h^{1}\left(\mathscr{Q}_{X}\right)=0$ by the same arguments as above. Furthermore, the vanishing also holds if $L=$ $B^{\otimes p+2+k} \otimes N$ and $L^{\prime}=B^{\otimes p+2+l} \otimes N^{\prime}$ for nef line bundles $N$ and $N^{\prime}$ on $X$.

Theorem 2.4 says in particular that $B^{\otimes n}$ satisfies property $N_{1}$, i.e., that the image of the embedding induced by $\left|B^{\otimes n}\right|$ is ideal-theoretically cut out by quadrics, if $h^{0}(B) \geq 5$ and $n \geq 3$. The bound imposed on $h^{0}(B)$ is sharp, since Example 1.5 provides an example in which $h^{0}(B)=4$ and $B^{\otimes 3}$ does not even satisfy property $N_{0}$ (cf. Theorem 1.4). We present now an example in which $B^{\otimes 3}$ satisfies property $N_{0}$, but not property $N_{1}$ :

Example 2.5. Let $X$ be a cyclic triple cover of $\mathbf{P}^{3}$ ramified along a smooth sextic surface and let $B$ be the pullback of $\mathscr{C}_{\mathbf{3}}(1)$ to $X$. The threefold $X$ is a Calabi-Yau threefold and $h^{0}(B)=4$. By Theorem 1.7, $B^{\otimes 3}$ satisfies property $N_{0}$. However, $B^{\otimes 3}$ does not satisfy property $N_{1}$.

Proof. We sketch the proof of the last claim. Assume $L=B^{\otimes 2}$ satisfies $N_{1}$. By Theorem 2.2 the assumption implies

$$
H^{1}\left(\bigwedge^{2} M_{L} \otimes L^{\otimes n}\right)=0
$$

for all $n \geq 1$. Let $S \in|B|$ and let $C$ be a smooth curve in $\left|B \otimes \mathscr{O}_{C}\right|$. Using (2.1) it can be seen that both $H^{2}\left(M_{L}^{\otimes 2} \otimes L^{\otimes n} \otimes B^{*}\right)$ and $H^{2}\left(M_{L}^{\otimes 2} \otimes L^{\otimes n} \otimes B^{*} \otimes \mathscr{C}_{S}\right)$ vanish. Those vanishings together with (2.5.1) imply that $H^{1}\left(\bigwedge^{2} M_{L} \otimes L^{\otimes n} \otimes \mathscr{O}_{C}\right)=0$. On the other hand there is an epimorphism between the vector bundles $M_{L} \otimes \mathscr{C}_{C}$ and $M_{L \otimes O_{C}}$ on $C$. Therefore we have

$$
H^{1}\left(\bigwedge^{2} M_{L \otimes \mathscr{C}_{C}} \otimes L^{\otimes n}\right)=0
$$

for all $n \geq 1$. It is a well known result by Castelnuovo that a line bundle of degree greater than or equal to $2 g+1$ on a smooth curve satisfies property $N_{0}$. The curve $C$ has genus 4 and $L \otimes \mathscr{Q}_{C}$ has degree 9, hence $L \otimes \mathscr{Q}_{C}$ satisfies $N_{0}$. 
Thus it would follow from (2.5.2) that $L \otimes \mathscr{O}_{C}$ satisfies also property $N_{1}$. But this contradicts a result by Green and Lazarsfeld (cf. [GL]), which says that a line bundle cannot satisfy property $N_{1}$ if it is the tensor product of the canonical bundle on $C$ and an effective line bundle of degree 3 , as is the case of $L \otimes Q_{C}$. Therefore the original assumption (2.5.1) is false and $L$ does not satisfies property $N_{1}$.

Remark 2.6. In Theorem 2.4 we dealt with the vanishings needed for property $N_{p}$, but in fact the arguments used yield more general cohomology vanishings: Let $X$ be a Calabi-Yau threefold and let $B$ be an ample and base-point-free line bundle such that $h^{0}(B) \geq 5$. Then $H^{1}\left(M_{B}^{\otimes n_{1}} \otimes \cdots \otimes M_{B} \otimes n_{p+1} \otimes B^{\otimes n}\right)=0$ for all $n \geq p+2, n_{1} \geq 3$ and $n_{2}, \ldots n_{p+1} \geq 1$.

We show now that the line bundle $L$ of Theorem 2.4 embeds the Calabi-Yau threefold as a variety with a Koszul coordinate ring.

Theorem 2.7. Let $X$ be a Calabi-Yau threefold. Let $B$ be an ample and base-pointfree line bundle with $h^{0}(B) \geq 5$. Let $L=B^{\otimes p+2+k}$ and $L^{\prime}=B^{\otimes p+2+l}$. If $k, l \geq 0$ and $p \geq 1$, then the coordinate ring of the image of the embedding induced by $|L|$ is Koszul.

Sketch of proof. We follow the same philosophy used in other proofs in this article. The claim follows from results regarding the Koszul property proven for surfaces of general type in [GP2]. Precisely it follows as a corollary of [GP2], Theorem 5.14, using [GP2], Lemma 3.4, and Observation 1.3 with the same strategy used to prove [GP2], Theorem 3.5.

As we did in Sect. 1, we obtain the following corollary for powers of ample line bundles. The proof is analogous to the proof of Corollary 1.10.

Corollary 2.8. Let X be a (smooth) Calabi-Yau threefold and let $A$ be an ample line bundle. Let $L=A^{\otimes n}$.

1. If $n \geq 4 p+8$, then L satisfies property $N_{p}$. Moreover, if $A^{3}>1$ and $n \geq 3 p+6$, then $L$ satisfies property $N_{p}$.

2. If $n \geq 12$ or if $A^{3}>1$ and $n \geq 9$, the coordinate ring of the image of the embedding induced by $\left|A^{\otimes n}\right|$ is Koszul.

\section{Appendix: Singular Calabi-Yau threefolds}

Throughout the previous part of this article we have been concerned only with smooth Calabi-Yau threefolds for reasons of simplicity. In this appendix we show that our arguments can be adapted without much difficulty to canonical CalabiYau threefolds and that our main theorems hold indeed for them.

There were only two instances in the proofs of Theorems 1.4 and 1.7 when the assumption of the nonsingularity of $X$ was used. The first of them was when 
we wanted to guarantee the vanishing of $H^{1}\left(B^{\otimes n}\right)$ for an ample line bundle $B$ and all $n \geq 0$. This vanishing holds as well for Calabi-Yau threefolds with canonical singularities. The second was to find, firstly a smooth surface $S$ in $|B|$, and secondly a smooth curve $C$ in $\left|B \otimes \odot_{S}\right|$. If $X$ has canonical singularities, it is not possible in general to find a smooth surface $S$ in $|B|$, since $S$ could have (at worst) rational double points, but it is possible to find a smooth curve $C$ in $\left|B \otimes Q_{S}\right|$ using a Bertini-type argument. Thus the only troublesome point is the use of Green's theorem. However this result can still be applied to $S$ general in $|B|$ if $X$ has canonical singularities, since we can apply it to the desingularization $\tilde{S}$ of $S$, for $S$ and $\tilde{S}$ have the same canonical ring. The upshot of all this is that Theorem 1.4, Theorem 1.7, and Theorem 2.4 hold for canonical CalabiYau threefolds, having only in account in the case of Theorem 1.7 that there is another case to add to Proposition 1.6 (6), namely the image of $X$ being a cone over a smooth 2-dimensional rational normal scroll.

As in the end of Sects. 1 and 2, we state now corollaries regarding powers of ample line bundles. We use for this purpose a generalization of Ein and Lazarsfeld's result on base-point-freeness, carried out by Oguiso and Peternell. They prove among other things (cf. [OP], Theorems I(2) and II(2)) that $A^{\otimes n}$ is base-point-free and gives a birational morphism if $n \geq 5$ and $A$ is an ample line bundle on a Calabi-Yau threefold with terminal singularities and if $n \geq 7$ and $A$ is an ample line bundle on a Calabi-Yau threefold with canonical singularities. As corollaries of their result and of [G2], Theorem 4, we recover their results on normal generation of powers of ample line bundles (see [OP], Theorems I(3) and II(3)) and generalize them to normal presentation and higher syzygies. We point out that [OP], Theorem 3 can also be recovered as corollary of our Theorem 1.7.

Corollary A.1 ([OP], Theorem I, (3)). Let X be a Calabi-Yau threefold with Qfactorial terminal singularities and let $A$ be an ample line bundle on $X$. If $n \geq 10$, then $A^{\otimes n}$ satisfies property $N_{0}$.

Corollary A.2 ([OP], Theorem II, (3)). Let $X$ be a Calabi-Yau threefold with canonical singularities and let $A$ be an ample line bundle on $X$. If $n \geq 14$, then $A^{\otimes n}$ satisfies property $N_{0}$.

As corollaries of Theorem 2.4, we obtain:

Corollary A.3. Let $X$ be a Calabi-Yau threefold with $\mathbf{Q}$-factorial terminal singularities and let $A$ be an ample line bundle on $X$. If $n \geq 5 p+10$, then $A^{\otimes n}$ satisfies property $N_{p}$. Furthermore, if $p \geq 1$, the coordinate ring of the image of the embedding induced by $\left|A^{\otimes n}\right|$ is Koszul.

Corollary A.4. Let $X$ be a Calabi-Yau threefold with canonical singularities and let $A$ be an ample line bundle on $X$. If $n \geq 7 p+14$, then $A^{\otimes n}$ satisfies property $N_{p}$. Furthermore, if $p \geq 1$, the coordinate ring of the image of the embedding induced by $\left|A^{\otimes n}\right|$ is Koszul. 


\section{References}

[ACGH] E. Arbarello, M. Cornalba, P.A. Griffiths, J. Harris, Geometry of Algebraic Curves, Volume I, Springer-Verlag 1985.

[B] D. Butler, Normal generation of vector bundles over a curve, J. Differential Geometry 39 (1994) $1-34$.

[C] C. Ciliberto, Sul grado dei generatori dell'anello canonico di una superficie di tipo generale, Rend. Sem. Mat. Univ. Politecn. Torino 41 (1983), 83-111.

[EH] D. Eisenbud, J. Harris, On varieties of minimal degree (A centennial account), Algebraic Geometry, Bowdoin 1985, Amer. Math. Soc. Symp. in Pure and App. Math. 46 (1987), $1-14$.

[EL1] L. Ein, R. Lazarsfeld, Koszul cohomology and syzygies of projective varieties, Invent. Math. 111 (1993), 51-67.

[EL2] L. Ein, R. Lazarsfeld, Global generation of pluricanonical and adjoint linear series on smooth projective threefolds, J. Amer. Math. Soc. 6 (1993), 875-903.

[F] T. Fujita, Remarks on Ein-Lazarsfeld criterion of spannedness of adjoint bundles on polarized threefolds, preprint.

[GP1] F.J. Gallego, B.P. Purnaprajna, Vanishing theorems and syzygies for K3 surfaces and Fano varieties, to appear in Journal of Pure and Applied Algebra.

[GP2] F.J. Gallego, B.P. Purnaprajna, Projective normality and syzygies of algebraic surfaces, preprint.

[G1] M. Green, The canonical ring of a variety of general type, Duke Math. J. 49 (1982), 1087-1113.

[G2] M. Green, Koszul cohomology and the geometry of projective varieties, J. Differential Geometry 19 (1984) 125-171.

[GL] M. Green, R. Lazarsfeld, Some results on the syzygies of finite sets and algebraic curves, Compositio Math. 67 (1989) 301-314.

[K] Y. Kawamata, On Fujita's freeness conjecture for 3-folds and 4-folds, Math. Ann. 308 (1997), 491-505.

[L] R. Lazarsfeld, A sampling of vector bundle techniques in the study of linear series, Lectures on Riemann Surfaces, World Scientific Press, Singapore, 1989, 500-559.

[Ma] A. Mayer, Families of K-3 surfaces, Nagoya Mathematics Journal 48 (1972), 1-17.

[Mi] Y. Miyaoka, The Chern class and Kodaira dimension of a minimal variety, Algebraic Geometry - Sendai 1985, Advanced Studies in Pure Math., Vol. 10, North-Holland, Amsterdam (1987), 449-476.

[Mu D. Mumford Varieties defined by quadratic equations, Corso CIME in Questions on Algebraic Varieties, Rome (1970), 30-100.

[OP] K. Oguiso, T. Peternell, On polarized canonical Calabi-Yau threefolds, Math. Ann. 301 (1995), 237-248.

[P] G. Pareschi, Gaussian maps and multiplication maps on certain projective varieties, Compositio Math. 98 (1995), 219-268.

[S-D] B. Saint-Donat, On projective models of K3 surfaces, Amer. J. Math. 96 (1974), 602-639. 